\title{
Estado nutricional e consumo alimentar de mulheres jovens na fase lútea e folicular do ciclo menstrual
}

\section{(} ORIGINAL | ORIGINAL

Nutritional status and food consumption of young women in the luteal and follicular phases of the menstrual cycle

Larissa Almenara Silva dos SANTOS 1

Clarissa SOARES ${ }^{1}$

Adriana Coutinho Giusti DIAS ${ }^{1}$

Nathália PENNA ${ }^{1,2}$

Antonio Orestes de Salvo CASTRO2

Vilma Blondet de AZEREDO ${ }^{1}$

\section{RE S U M O}

\section{Objetivo}

Avaliar o estado nutricional e a ingestão alimentar de mulheres adultas sadias durante o ciclo menstrual.

\section{Métodos}

Quarenta e cinco voluntárias foram acompanhadas durante três meses. A avaliação do estado nutricional foi baseada no índice de massa corporal, porcentagem de gordura e água corporal. Foram aplicados seis registros alimentares para análise da ingestão dos grupos de alimentos, usando como base o guia alimentar da pirâmide. Para a observação do sintoma "desejos alimentares", foram utilizados três "mapas de sintomas diários".

\section{Resultados}

Os valores médios de índice de massa corporal e de porcentagem de gordura corporal apresentaram-se normais em ambas as fases, entretanto foi observado maior percentual de mulheres com água corporal acima do padrão na fase lútea (77\%). O consumo de alimentos do grupo complementar foi maior na fase lútea. Todos os outros grupos de alimentos, com exceção do grupo de carnes, apresentaram consumo inferior às recomendações, em ambas as fases. A intensidade do sintoma "desejos alimentares" foi leve durante o ciclo menstrual, não sendo observada diferença significativa entre as fases. O sintoma "desejos alimentares" associou-se positivamente com o aumento da ingestão do grupo complementar na fase lútea.

\footnotetext{
${ }^{1}$ Universidade Federal Fluminense, Faculdade de Nutrição, Departamento de Nutrição e Dietética. R. Mário Santos Braga, 30, $4^{\circ}$ andar, Praça do Valonguinho, 24020-140, Niterói, RJ, Brasil. Correspondência para/Correspondence to: V.B. AZEREDO. E-mail:<vilma.blondet@predialnet.com.br>.

${ }^{2}$ Universidade Estadual do Rio de Janeiro, Instituto de Matemática e Estatística. Rio de Janeiro, RJ, Brasil.
} 
324 | L.A.S. SANTOS et al.

\section{Conclusão}

O ciclo menstrual influencia o comportamento alimentar e leva à retenção hídrica, na fase lútea, em mulheres adultas sadias.

Termos de indexação: Ciclo menstrual. Consumo alimentar. Estado nutricional. Síndrome pré-menstrual.

\section{A B S T R A C T}

\section{Objective}

This study assessed the nutritional status and food intake of healthy young women during the menstrual cycle.

\section{Methods}

Forty-five volunteers were followed for three months. Their nutritional status was determined by body mass index, body fat and total body water. Six food recall instruments were used to evaluate food group intake according to the food guide pyramid. Food cravings were detected by three daily symptom charts.

\section{Results}

The mean body mass index and body fat were normal in both phases; however, there was a higher percentage of women with above-average body water during the luteal phase (77\%). The consumption of foods from the complementary group was higher during the luteal phase. The consumption of foods from all other groups during both phases was below the recommended levels, except for meats. Food cravings were mild during the entire menstrual cycle and there were no significant differences between the phases. Food cravings were positively associated with increased intake of foods from the complementary group.

\section{Conclusion}

In healthy women, the menstrual cycle influences food consumption and the luteal phase causes water retention.

Indexing terms: Menstrual cycle. Food consumption. Nutritional status. Premenstrual syndrome.

\section{N T R O D U ÇÃ O}

Pesquisas relacionam mudanças no comportamento alimentar, no tocante à quantidade e à qualidade de alimentos consumidos durante as fases do ciclo menstrual, especialmente na fase lútea $^{1-3}$. Entretanto, a maioria dos estudos baseia-se apenas nas alterações ocorridas em mulheres com Síndrome Pré-Menstrual (SPM). Embora muitas mulheres sofram com a sintomatologia relacionada à SPM, apenas 2\% a 3\% são gravemente afetadas, o que leva à necessidade de atendimento à sintomatologia da paciente.

De acordo com a National Association For Premenstrual Syndrome ${ }^{4}$, mais de $90 \%$ das mulheres que menstruam experimentam alguma mudança pré-menstrual, e entre $5 \%$ a $10 \%$ das mulheres no Reino Unido são severamente incapacitadas de dominar sua vida durante essa fase do ciclo - sendo a SPM severa mais comum entre os 30 e 40 anos de idade. Permanece desconhecida a etiologia e a fisiodoença da SPM ${ }^{5}$, acredita-se que seja provavelmente um resultado de severas mudanças fisiológicas que envolvem hormônios ovarianos, mineralocorticóides, prolactina, androgênios, prostaglandinas, fatores nutricionais - por exemplo, piridoxina e ácidos graxos essenciais -, hipoglicemia, dentre outros ${ }^{4}$. Os sintomas são cíclicos e recorrentes ${ }^{6}$. Alguns sintomas físicos são conhecidos: dor de cabeça, enxaqueca, dores e inchaço articular e muscular, dores nas costas (principalmente lombar) e "desejo" por determinados alimentos?.

No Brasil, são escassos os estudos que relacionam a alimentação com os sintomas da SPM. Assim, a fim de contribuir com a melhoria da abordagem nutricional e diante dos aspectos que fazem parte da fisiologia feminina, este estudo pretende avaliar a ingestão de grupos de alimentos e o estado nutricional de mulheres em idade reprodutiva durante o ciclo menstrual e observar as possíveis relações entre a ingestão alimentar e o sintoma "desejos alimentares" relacionado com a SPM. 


\section{MÉ T O D O S}

Este é um estudo do tipo longitudinal, aleatorizado, que realizou o acompanhamento de mulheres jovens, estudantes, por um período de três meses. Utilizamos como critério de seleção para participação no projeto, o ciclo menstrual regular (25 a 35 dias), a idade mínima de 20 anos e máxima de 40 anos, a ausência de doenças, de tabagismo e do uso de suplementos nutricionais. Este intervalo de idade foi escolhido pelo fato da mulher estar em idade reprodutiva e sem, prováveis, alterações hormonais ocasionadas em idades inferiores (adolescência) ou superiores a esta.

O recrutamento das voluntárias $(n=45)$ e o desenvolvimento do estudo foram realizados na Faculdade de Nutrição da Universidade Federal Fluminense (UFF), no Município de Niterói, durante $\mathrm{o}$ ano de 2008. As atividades foram iniciadas somente após aprovação do protocolo de estudo pelo Comitê de Ética responsável por estudos em humanos do Hospital Universitário Antônio Pedro, da UFF. Um consentimento de participação na pesquisa foi assinado pelas voluntárias, após esclarecimentos da finalidade do estudo e do uso dos dados sob a garantia de anonimato. Uma vez cientes e de acordo com a proposta da pesquisa, as voluntárias foram entrevistadas para a caracterização do grupo e para a coleta de informações sobre variáveis demográficas, história patológica pregressa, dados antropométricos, estilo de vida, menarca e início de relações sexuais. O questionário padronizado foi aplicado pelo pesquisador responsável na primeira abordagem em função do aceite em participar do estudo.

A avaliação da ingestão alimentar das voluntárias foi realizada pela aplicação de seis registros alimentares: 3 na fase lútea (do $23^{\circ}$ ao $25^{\circ}$ dia do ciclo menstrual) e 3 na fase folicular (do $7^{\circ}$ ao $9^{\circ}$ dia do ciclo menstrual), mensalmente, totalizando 18 registros por voluntária. As voluntárias foram devidamente orientadas quanto ao preenchimento dos registros alimentares. O cálculo de ingestão dos grupos de alimentos foi realizado considerando o Guia Alimentar da Pirâmide adaptada para a população brasileira e com base no seu porcionamento ${ }^{8}$. Os grupos alimentares analisados foram: 1) cereais, tubérculos, raízes e seus derivados; 2) hortaliças; 3) frutas; 4) leite e derivados; 5) carnes e ovos; 6) leguminosas; 7) complementar. Esse último grupo refere-se a alimentos e bebidas com alto teor de gorduras, açúcares e sal, considerados prejudiciais à saúde quando consumidos de maneira indiscriminada e em quantidades acima da recomendada.

A avaliação antropométrica das voluntárias envolveu a medição de seu peso (massa corporal em quilos) e estatura (em metros). Essas medidas foram utilizadas para o cálculo do Índice de Massa Corporal (IMC): massa corporal, em quilos, dividida pela estatura, em metro, elevada ao quadrado.

A avaliação antropométrica foi realizada no mesmo período da avaliação dietética: na fase lútea (do $23^{\circ}$ ao $25^{\circ}$ dia do ciclo) e na fase folicular (do $7^{\circ}$ ao $9^{\circ}$ dia do ciclo). Para a aferição do peso corporal, percentual de gordura e água corporal total, as voluntárias estavam em jejum de 12 horas e sem praticar atividade física nas últimas 24 horas. Na pesagem, as voluntárias estavam descalças, com roupão leve (cirúrgico) em posição ortostática, em plano Frankfurt, sendo a leitura feita em quilogramas. Para avaliação da composição corporal, foi utilizada balança de bioimpedância (BIA) Tanita, modelo BC - 418 - precisão de 0,1 kg, tetrapolar, com sensores para a planta dos pés e das mãos, do laboratório de Avaliação Nutricional e Funcional da Faculdade de Nutrição da UFF (LANUFF); a estatura foi obtida por meio de estadiômetro.

Para classificar o estado nutricional das voluntárias, baseado no IMC, foram adotados os critérios da Organização Mundial de Saúde (OMS)9: Baixo peso, IMC <18,4; eutrofia, IMC entre 18,5 - 24,9; sobrepeso, IMC entre 25,0 - 29,9; obesidade, $I M C>30 \mathrm{~kg} / \mathrm{m}^{2}$. Para avaliação da composição corporal, no que diz respeito ao percentual de gordura para mulheres, foi considerado intervalo de normalidade entre $25 \%$ a $30 \%{ }^{10}$. Para avaliação da adequação do percentual de água 
corporal, foi utilizado como referência o valor médio de $50 \%$ da massa corporal11 ${ }^{11}$.

O sintoma "desejos alimentares" relacionado à síndrome pré-menstrual foi avaliado a partir da aplicação de um "diário" apropriado (Daily Symptom Report) ${ }^{7}$, no qual as voluntárias assinalaram diariamente, durante três meses, o sintoma identificado. Esse sintoma recebeu um escore em uma escala de cinco pontos: $0=$ ausente; 1 = leve (apenas levemente aparente); 2 = moderado (o sintoma é perceptível, mas não altera a rotina diária); 3 = alto (continuamente incomodada pelo sintoma e/ou o sintoma interfere na atividade diária); 4 = grave (o sintoma é maior do que se pode controlar/suportar e/ou impossibilita o prosseguimento da atividade diária). O registro de sintomas diários (Daily Symptom Report) vem sendo bastante utilizado e compõe-se de uma lista de 17 sintomas de ocorrência comum no desconforto pré-menstrual: irritabilidade, choro, cansaço, mudanças de humor, compulsão para consumo de alimentos, tensão, seios doloridos, ansiedade, inchaço, depressão, cólica, sensação de perda de controle, dores contínuas, má coordenação, cefaleia, confusão e insônia².

Os resultados, no presente estudo, são apresentados por meio da estatística descritiva, como média aritmética e erro-padrão. Análise de variância (ANOVA one-way) foi utilizada para análise longitudinal do estado nutricional, da ingestão de grupos de alimentos e da intensidade do sintoma "desejos alimentares". Como pós-teste foi utilizado o teste de Tukey. Para a comparação das médias nos diferentes períodos do ciclo menstrual (fase lútea e folicular), foi aplicado o teste $t$-pareado. Correlação de Pearson foi utilizada para verificar possíveis associações entre a ingestão de grupos de alimentos e o desejo alimentar no ciclo menstrual. Foi utilizado o programa Statistical Package for the Social Sciences (SPSS) for Windows ${ }^{12}$, versão 14.0 , para a realização das diferentes análises, tendo sido aceito um nível de significância de $p<0,05$. A suposição de normalidade (distribuição Gaussiana) dos dados foi verificada pelos testes de Kurtosis e Skewness, para suportar a utilização dos métodos estatísticos descritos acima.

O protocolo de pesquisa cumpriu os princípios éticos contidos na Declaração de Helsinki e as normas da resolução 196/96 do Conselho Nacional de Saúde, tendo sido aprovado pelo Comitê de Ética em Pesquisa com Seres Humanos do Hospital Universitário Antonio Pedro, constando aprovado no Sistema Nacional de Informação sobre Ética em Pesquisa envolvendo Seres Humanos (SISNEP) sob o protocolo número 0084.0.258.00 0 -07. Todos os participantes assinaram um termo de consentimento livre e esclarecido antes de sua inclusão na amostra.

\section{RESULTADOS}

A maioria das voluntárias eram estudantes universitárias solteiras, com renda familiar média de nove salários-mínimos. A idade Média (M) das voluntárias foi de 23,20 Erro-Padrão $(E P)=0,61$ anos, com a menarca aos $M=12,10, E P=0,19$ anos. O início da relação sexual aconteceu ao redor de 18 anos. Apenas 41,0\% das voluntárias relataram prática de atividade física regular em academia ( $M=2,44, E P=0,74$ horas/semana), enquanto a maioria $(59,0 \%)$ não desempenhava nenhum tipo de atividade física relacionada a esporte e lazer, somente atividades acadêmicas na universidade. Cerca de $44,0 \%$ das voluntárias utilizavam unicamente o método contraceptivo hormonal; 9,0\% utilizavam o método de barreira (camisinha); $23,5 \%$ utilizavam os dois métodos, e $23,5 \%$ não faziam uso de nenhum método contraceptivo. O ciclo menstrual, em média, tinha duração de 28,20, EP=0,33 dias, podendo ser considerado normal ${ }^{13}$.

Os valores representam a média dos três meses de acompanhamento. Com relação à massa corporal total e ao IMC, observou-se que, em média, a maioria (>80,0\%) apresentava-se eutrófica, não havendo alteração significativa do estado nutricional ao longo do estudo e entre as duas fases do ciclo. Foi observado apenas um caso de baixo peso $(2,9 \%)$ e pouco mais de $14,0 \%$ das 
voluntárias apresentavam IMC dentro dos valores acima da normalidade (sobrepeso e/ou obesidade). Os valores médios do percentual de gordura observados neste estudo nas fases lútea e folicular foram considerados normais, estando em adequação ao longo do estudo. Todavia, 14,7\% das voluntárias na fase lútea e $17,6 \%$ na fase folicular tinham sua gordura corporal acima de $30,0 \%$. A quantidade de massa livre de gordura não apresentou alteração significativa durante o estudo (Tabela 1).

A concentração média de água corporal não apresentou diferença estatística significativa da fase lútea para a fase folicular. No entanto, ao observar a frequência de adequação desse indicador em cada fase, constatou-se que na fase lútea a maioria das voluntárias $(77,0 \%)$ apresentava a água corporal acima do padrão de referência $(>50,0 \%)$, enquanto na fase folicular foi observada menor frequência $(54,0 \% ; p<0,05)$ (Figura $1 \mathrm{~A}$ e B). Ao se verificar a possível associação entre o uso de contraceptivo hormonal e retenção hídrica, pôde-se observar que a utilização desse método contraceptivo não influenciou o percentual de água corporal.

Como esperado, ao relacionar a gordura corporal com o IMC, observou-se correlação positiva significativa entre o IMC e o aumento do percentual de gordura corporal $(r=0,8248 ; p=0,023)$. Ainda foi observada uma associação negativa significativa entre a água corporal e o IMC $(r=-0,8546 ; p=0,001)$ : quanto maior o IMC e, consequentemente, a gordura corporal, menor o percentual de água corporal.

A ingestão de alimentos foi categorizada por grupos e em porções (Tabela 2). Em média, as voluntárias realizavam quatro refeições diárias tanto na fase lútea quanto na folicular. Não houve diferença estatística significativa no consumo entre a maioria dos grupos de alimentos no ciclo menstrual, exceto para o grupo de alimentos complementares que teve o consumo aumentado $(p=0,04)$ na fase lútea.

Ao comparar a ingestão média dos grupos de alimentos com a recomendação do Guia Ali-
Tabela 1. Composição corporal das voluntárias nas diferentes fases do ciclo menstrual. Niterói (RJ), 2008.

\begin{tabular}{lccccc}
\hline \multirow{2}{*}{ Indicador } & \multicolumn{2}{c}{ Lútea } & & \multicolumn{2}{c}{ Folicular } \\
\cline { 2 - 3 } \cline { 6 - 7 } & $\mathrm{M}$ & $\mathrm{EP}$ & & $\mathrm{M}$ & $\mathrm{EP}$ \\
\hline Gordura corporal (\%) & 27,54 & 1,46 & & 27,92 & 1,67 \\
Massa livre de gordura (\%) & 32,96 & 1,02 & & 32,48 & 0,90 \\
Água corporal (\%) & 51,57 & 0,95 & & 50,92 & 1,19 \\
Peso corporal (kg) & 60,50 & 4,03 & & 60,40 & 5,10 \\
IMC $\left(\mathrm{kg} / \mathrm{m}^{2}\right)$ & 22,90 & 0,65 & & 22,60 & 0,80 \\
\hline
\end{tabular}

*Valores representam a média dos três meses de acompanhamento. Não foi observada variação significativa no estado nutricional ao longo do estudo; M: média; EP: erro-padrão.

A

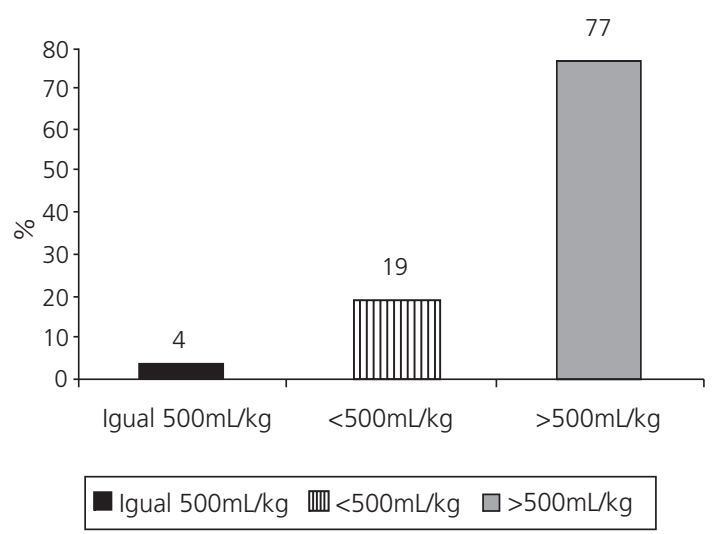

B

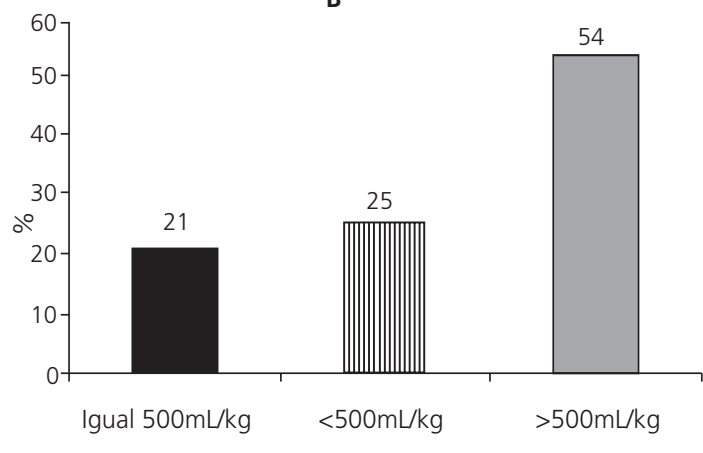

Igual $500 \mathrm{~mL} / \mathrm{kg}$ 血 $<500 \mathrm{~mL} / \mathrm{kg} \quad \square>500 \mathrm{~mL} / \mathrm{kg}$

Figura 1. Frequência média de adequação da água corporal na fase lútea (A) e folicular (B) do ciclo menstrual. Niterói (RJ), 2008

Nota: Não foi observada variação significativa deste indicador ao longo do estudo (3 meses).

mentar da Pirâmide ${ }^{8}$, observou-se baixa ingestão para a maioria dos grupos durante o ciclo menstrual, enquanto apenas o grupo de carnes apre- 
sentou adequação em ambas as fases. O grupo de alimentos complementar na fase folicular, em média, teve adequação de consumo, atingindo a recomendação, e, na fase lútea, apresentou um consumo excessivo (Tabela 2). Apresenta-se a frequência de ingestão de alimentos do grupo complementar acima da recomendação sugerida pelo guia alimentar, podendo ser observada maior frequência $(43,0 \%)$ de consumo excessivo ( $\geq 5$ porções) na fase lútea do ciclo menstrual, em relação à fase folicular (33,0\%) (Figura 2).

Em média, em ambas as fases do ciclo menstrual, observou-se a presença do sintoma "desejos alimentares", com intensidade leve (fase lútea: $M=0,78 E P=0,15$; fase folicular: $M=0,67$ $E P=0,13)$, não havendo diferença estatística significativa. No entanto, a maior frequência $(7,4 \%)$ de voluntárias com sintomatologia de alta intensidade (nível 3) para "desejos alimentares" foi observada na fase lútea do ciclo, enquanto na fase folicular essa frequência caiu para 3,7\%. Não houve relato da sintomatologia grave (4) tanto na fase lútea quanto na folicular.

A Figura 3 exibe a oscilação do sintoma "desejos alimentares" no decorrer do ciclo menstrual. Sua maior intensidade se dá na fase lútea do ciclo - por volta do $22^{\circ}$ dia do ciclo -, quando esse sintoma começa a aumentar e se intensificar, tendo seu ápice nos dias que antecedem o sangramento, quando apresenta sua intensidade máxima. Depois do sangramento, esse sintoma diminui (fase folicular), e do $2^{\circ}$ dia em diante sua intensidade cai, estabilizando-se entre o décimo e o décimo nono dia, aproximadamente, quando torna a aumentar. Esses sintomas foram observados nos três ciclos estudados, não havendo variação significativa entre os meses. Observou-se associação positiva e significativa $(r=0,50$ e $p=0,0097)$ entre o sintoma "desejos alimentares" e o maior consumo de alimentos do grupo complementar.

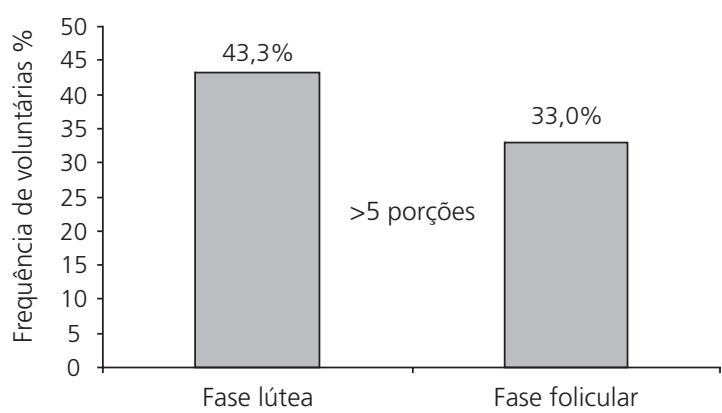

Figura 2. Frequência de voluntárias com consumo de alimentos do grupo complementar acima de 5 porções/dia. Niterói (RJ), 2008.

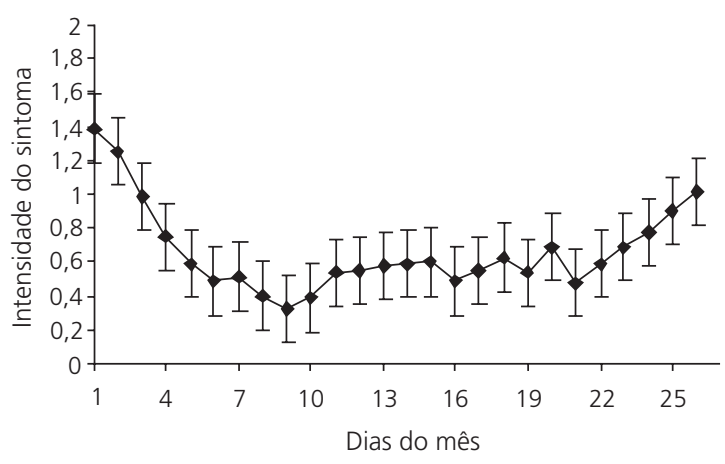

Figura 3. Flutuação do sintoma "desejos alimentares" durante o ciclo menstrual. Niterói (RJ), 2008.

Tabela 2. Consumo alimentar médio, diário, dos grupos de alimentos (em porções). Niterói (RJ), 2008.

\begin{tabular}{|c|c|c|c|c|c|c|c|c|c|}
\hline \multirow{2}{*}{ Grupos de alimentos } & \multirow{2}{*}{$\begin{array}{l}\mathrm{N}^{\circ} \text { de porções } \\
\text { recomendadas }\end{array}$} & \multicolumn{2}{|c|}{ Lútea } & \multicolumn{2}{|c|}{ Folicular } & \multicolumn{2}{|c|}{ Lútea } & \multicolumn{2}{|c|}{ Folicular } \\
\hline & & M & EP & $\mathrm{M}$ & $\mathrm{EP}$ & Mínimo & Máximo & Mínimo & Máximo \\
\hline Cereais & $5-9$ & 3,0 & 0,12 & 2,9 & 0,17 & 1,7 & 4,4 & 1,0 & 4,8 \\
\hline Hortaliças & $4-5$ & 1,0 & 0,16 & 0,8 & 0,13 & 0,0 & 3,2 & 0,5 & 3,2 \\
\hline Frutas & $3-5$ & 1,2 & 0,18 & 1,4 & 0,23 & 0,0 & 3,4 & 0,0 & 4,6 \\
\hline Leite & 3 & 1,7 & 0,15 & 1,6 & 0,12 & 0,3 & 3,8 & 0,3 & 2,8 \\
\hline Carnes & $1-2$ & 1,7 & 0,12 & 1,7 & 0,12 & 0,0 & 3,1 & 0,0 & 2,6 \\
\hline Leguminosas & 1 & 0,4 & 0,07 & 0,5 & 0,07 & 0,0 & 1,9 & 0,0 & 1,2 \\
\hline Complementares & $1-4$ & $5,0^{*}$ & 0,45 & 4,0 & 0,34 & 1,7 & 13,3 & 0,6 & 9,1 \\
\hline
\end{tabular}

*Valores significativamente diferentes entre colunas; M: média; EP: erro-padrão. 


\section{DIS CUSS Ã O}

O este estudo demonstra que as mulheres adultas estudadas apresentam prática alimentar inadequada, com baixo consumo de alimentos, principalmente, dos grupos de frutas e hortaliças. Dados nacionais do Instituto Brasileiro de Geografia e Estatística ${ }^{14}$ confirmam esses resultados ao mostrar a diminuição no consumo de alimentos tradicionais da dieta do brasileiro, como o arroz e o feijão. Por outro lado, o consumo de produtos industrializados, como biscoitos e refrigerantes, aumentou em $400 \%$. Na dieta das regiões metropolitanas, foi observado um excessivo consumo de açúcar, aumento de gorduras totais e saturadas e insuficiente consumo de frutas e hortaliças, corroborando os resultados encontrados no presente estudo.

Segundo alguns pesquisadores, o ciclo ovariano pode ocasionar mudanças emocionais, comportamentais, físicas e cognitivas em algumas mulheres, enquanto para outras essas manifestações passam despercebidas ${ }^{15,16}$. A literatura mostra modificações no comportamento alimentar de mulheres com síndrome pré-menstrual2 ${ }^{2}$, contudo existem poucas informações disponíveis, aqui no Brasil, sobre essas modificações durante o ciclo menstrual de mulheres sadias. O presente estudo observou maior consumo de alimentos do grupo complementar (doces, açúcares, óleos e gorduras) na fase lútea do ciclo menstrual. Esses resultados foram corroborados por Costa et al. ${ }^{3}$, em um estudo semelhante realizado em Santa Catarina com mulheres adultas. Esses autores observaram que as mulheres estudadas sentiam mais vontade de comer alimentos doces na fase lútea do ciclo. Outros estudos mostram que algumas mulheres que apresentam SPM aumentam a ingestão de alimentos açucarados e gordurosos durante a fase pré-menstrual 7,17 e que uma, entre três mulheres, apresenta aumento da fome e o desejo por alguns alimentos durante as duas semanas que antecedem o seu período menstrual, o que pode levar a um aumento na ingestão de calorias de até $87 \%$ nesse período ${ }^{2}$. Reed et al. ${ }^{2}$, ao comparar mulheres com SPM e mulheres normais em Nova York, observaram que as mulheres com SPM apresentavam maior intensidade de "desejos alimentares" e consumiam mais calorias na fase lútea do que as mulheres normais.

Pesquisadores mostram que após a ingestão de alimentos ricos em carboidratos, provenientes de alimentos açucarados e doces, a mulher apresenta alívio de sintomas como a depressão, tensão, confusão, tristeza e fadiga, sentindo-se mais calmas e alertas. Parece que esse aumento no consumo de carboidratos relaciona-se a uma tentativa de elevar os níveis de serotonina de modo a diminuir os efeitos negativos no humor ${ }^{7,17}$.

Sampaio ${ }^{18}$, em seu estudo de revisão, enfatiza a existência da relação entre o ciclo menstrual e alterações no comportamento alimentar, sendo um dos aspectos citados na literatura a flutuação de peso como conseqüência do aumento da ingestão energética na fase lútea, devido ao aumento do apetite, provocada pela oscilação hormonal. Apesar de, no presente estudo, ter sido observado aumento do consumo de alimentos do grupo complementar na fase lútea, não foi observada alteração significativa no peso corporal e, consequentemente, no IMC das voluntárias. Entretanto, houve uma sutil elevação no percentual de gordura na fase folicular, o que pode ter ocorrido pelo aumento de ingestão acima relatado (Tabela 1).

Quanto ao sintoma "desejos alimentares", observou-se uma elevação a partir do $22^{\circ}$ dia do ciclo menstrual, com intensidade máxima no primeiro dia do ciclo (no sangramento), o que é corroborado por estudos semelhantes realizados em outros países ${ }^{19-21}$. Possivelmente, esse fato tenha influenciado a média da intensidade do sintoma na fase folicular, não permitindo a observação de diferença significativa entre as duas fases, no presente estudo (Figura 3). Associação positiva desse sintoma com o aumento do consumo de alimentos do grupo complementar foi observada no presente estudo, porém não ocasionou um ganho de peso corporal significativo. Andrzej \& Diana ${ }^{22}$, enfatizam que, apesar da etiologia desses sintomas ainda não estar bem estabelecida, parece que os hormônios esteróides produzidos pelo corpo lúteo no ovário provocam os sintomas. Entretanto, no estudo de Dye et al. ${ }^{23}$, 
que analisaram os padrões de desejos alimentares e suas relações com o estado emocional, foi observada uma tendência à depressão nesse período do ciclo menstrual, justificando-se pela diminuição do nível de serotonina cerebral. Outros pesquisadores corroboram esses resultados, mostrando que os desejos alimentares nesse período podem ocorrer por meio de um mecanismo psicológico: as mulheres tentam reagir contra a depressão e o estresse, procurando na alimentação uma forma de conforto - ou por reação fisiológica -, buscando aumentar o nível de serotonina ${ }^{3}$. Não existe, portanto, um consenso na literatura a cerca da etiologia desse sintoma.

Quanto à retenção hídrica, estudos mostram que a alteração do nível de progesterona associada à fase lútea pode levar à retenção de líquidos e de sódio, ocasionando aumento do volume plasmático logo após a ovulação, alcançando valor máximo dois dias antes do sangramento ${ }^{24,25}$. No presente estudo, pode-se observar um grande número de voluntárias com percentual de água corporal acima do padrão $(>500 \mathrm{~mL} / \mathrm{kg})$ na fase lútea do ciclo. Cabe ressaltar que não foi observado efeito do uso de contraceptivo oral sobre o conteúdo de água corporal total nas mulheres estudadas, sugerindo a ação de outro fator para retenção hídrica. Costa et al. ${ }^{3}$, observaram resultado semelhante em seu estudo: mais de $70 \%$ das voluntárias apresentaram retenção hídrica nesse mesmo período. Segundo Novotny ${ }^{16}$, sintomas como ganho de peso, dor articular e cefaléia estão vinculados à retenção de líquidos, o que pode levar ao ganho temporário de peso.

De maneira geral, a literatura científica mostra o que empiricamente muitas mulheres já experimentaram: aumento do consumo alimentar, desejo por chocolate e alimentos ricos em açúcar simples durante a fase lútea, juntamente com sentimentos de estresse, ansiedade e tristeza, sendo esses sintomas bastante acentuados nas mulheres portadoras da SPM 2,18 e mais brandos em mulheres não portadoras da síndrome².

De acordo com o Colégio Americano de Ginecologia e Obstetrícia, os critérios para diagnóstico da SPM é feito apenas quando, ao menos, um sintoma mental e um somático ocor- rem em nível moderado ou intenso na fase lútea do ciclo. No presente estudo, não foi observada elevação na intensidade do sintoma moderado (3), e a presença do sintoma intenso (4) na fase lútea, o que impossibilitou a classificação das voluntárias aqui estudadas como portadoras da SPM.

Uma das vertentes para o tratamento da sintomatologia relacionada à SPM é a prática de atividade física, entretanto observamos no presente estudo que as mulheres estudadas apresentavam atividade muito leve ( $\leq 2$ horas/semana). Segundo Fernandes et al. ${ }^{26}$, a mulher com sintomatologia relacionada a SPM deve ser encorajada a fazer exercícios aeróbicos pelo menos três vezes por semana, principalmente na fase lútea, com o intuito de minimizar os sintomas. De acordo com Somer ${ }^{7}$, uma variedade de fatores no estilo de vida pode agravar os sintomas, incluindo o estresse, pouca atividade física, dieta rica em açúcar e carboidratos refinados, sal, gordura saturada, álcool e cafeína ${ }^{7}$.

\section{CONCLUSÃO}

Os resultados obtidos no presente estudo evidenciam que as mulheres estudadas apresentam ingestão deficiente da maioria dos grupos de alimentos nas duas fases do ciclo menstrual, o que pode ser um indicador de maus hábitos alimentares e de que o ciclo menstrual interfere no comportamento alimentar, aumentando a ingestão de alimentos doces, açucarados e gordurosos. Contudo, apesar de seus hábitos não salutares, a maioria das voluntárias apresenta adequado estado nutricional; a maior mudança encontrada foi no compartimento de água, o que levou ao aumento da água corporal na fase lútea do ciclo menstrual.

\section{A GRADECIMENTOS}

Às voluntárias participantes do estudo, à Pró-reitoria de Pesquisa e Pós-graduação da UFF e ao CNPq, pela concessão de bolsa de iniciação científica, e à FAPERJ. 


\section{COLABORADORES}

L.A.S SANTOS, C. SOARES, A.C.G. DIAS e N. PENNA responsáveis pelo levantamento bibliográfico, captação das voluntárias e organização dos dados. A.O.S. CASTRO descreveu o método estatístico e realizou as referidas análises. V.B. AZEREDO participou da concepção e delineamento e do método do estudo. Todos os autores participaram da redação do artigo.

\section{REFERÊ N CIAS}

1. Martini MC, Lampe JW, Slavin JL, Kurzer MS. Effect of the menstrual cycle on energy and nutrient intake. Am J Clin Nutr. 1994; 60:895-9.

2. Reed SC, Levin FR, Evans SM. Changes in mood, cognitive performance and appetite in the late luteal and follicular phases of the menstrual cycle in women with and without PMDD (premenstrual dysphoric disorder). Hormon Behav. 2008; 54:185-93.

3. Costa YR, Fagundes RLM, Cardoso BR. Ciclo menstrual e consumo de alimentos. Rev Bras Nutr Clín. 2007; 22(3):203-9.

4. National Association for Premenstrual Syndrome. Guide to premenstrual syndrome. London; 2007 [cited 2008 April 17]. Avaiable from: <http://www. pms.or.uk/Publications/>.

5. Ismail KMK, O’Brien S. Premenstrual syndrome. Curr Obstet Gynaecol. 2005; 15:25-30.

6. Silva CML, Gigante DP, Arret MLV, Fassa AG. Estudo populacional de sídrome pré-menstrual. Rev Saúde Pub. 2006; 40(1):47-56.

7. Somer E. Food An Owl Book Mood: the complete guide to eating well and feeling your best. $2^{\text {nd }}$ ed. New York: An Owl Book; 1999.

8. Phillips ST, Latterza AR, Cruz ATR, Ribeiro LC. Pirâmide alimentar adaptada: guia para escolha dos alimentos. Rev Nutr. 1999; 12(1):65-80. doi: 10.15 90/S1415-52731999000100006.

9. Organización Mundial de la Salud. El estado físico: uso y interpretacion de la antropometria. OMS: série de informes técnicos 854. Informe de un comite de expertos de la OMS. Genebra: OMS; 1995.

10. Lohman TG, Roche AF, Martorell R. Anthropometric standardization reference manual. Champaign: Human Kinetics Books; 1988.

11. Schoeller AD. Hydrometry. In: Heymsfield SB, Lohman TG, Wang Z, Going SB. Human body composition. $2^{\text {nd }}$ ed. Champaign: Human Kinetics; 2005. Chapter 3.

12. Statistical Package for the Social Science for Windows, version 14.0. Chicago (IL): SPSS Inc; 2005.
13. Guyton AC, Hall JE. Tratado de fisiologia médica. $10^{a}$ ed. Rio de Janeiro: Guanabara Koogan; 2002.

14. Instituto Brasileiro de Geografia e Estatística. Pesquisa de orçamentos familiares 2002-2003: primeiros resultados-Brasil e grandes regiões. Rio de Janeiro: IBGE; 2004.

15. Thys-Jacobs S, Starkey P, Bernstein D, Tian J. Calcium carbonate and the premenstrual syndrome: effects on premenstrual and menstrual symptoms. Am J Obstet Gynecol. 1998; 179(2): 444-52.

16. Novotny PP. Síndrome pré-menstrual (SPM): como tratar e evitar. Rio de Janeiro: Ediouro;1994.

17. Cross GB, Marley J, Miles H, Wilson K. Changes in nutrient intake during the menstrual cycle of overweight women with premenstrual syndrome. Br J Nutr. 2001; 85(4):475-82.

18. Sampaio HAC. Aspectos nutricionais relacionados ao ciclo menstrual. Rev Nutr. 2002; 15(3):309-17. doi: 10.1590/S1415-52732002000300007.

19. Rock CL, Gorenfo DW, Drewnowski A, Demitrack MA. Nutritional characteristics, eating pathology and hormonal status young women. Am J Clin Nutr. 1996; 64(4):566-71.

20. Dye L, Blundell JE. Menstrual cycle and appetite control: implications for weight regulation. Hum Reprod. 1997; 12(6):1142-51.

21. Kuga M, Ikeda M, Suzuki K. Gustatory changes associated with the menstrual cycle. Physiol Behav. 1999; 66(2):317-22.

22. Andrzej M, Diana J. Premenstrual syndrome: from etiology to treatment. Eur Menopause J. 2006; 55(s):s47-s54.

23. Dye L, Warner P, Bancroft J. Food craving during the menstrual cycle and its relationship to stress, happiness and depression; a premlimiray enquiry. J Affect Disord. 1995; 34(3):157-64.

24. Frankovit RJ, Lebrum CM. The athletic woman: menstrual cycle, contraception, and performance. Clin Sports Med. 2000; 19(2):251-71.

25. Bäckström T, Andreen L, Birzniece $V$, Björn I, Johansson IM, Nordenstam-Haghjo M, et al. The role of hormones and hormonal treatments in premenstrual syndrome. CNS Drugs. 2003; 17(5): 325-42.

26. Fernandes CE, Ferreira JAS, Azevedo LH, Pellini EAJ, Peixoto S. Síndrome da tensão pré-menstrual: o estado atual dos conhecimentos. Arq Med ABC. 2004; 29(2):77-81.

Recebido em: 22/6/2009

Versão final reapresentada em: 5/8/2010

Aprovado em: 19/10/2010 
